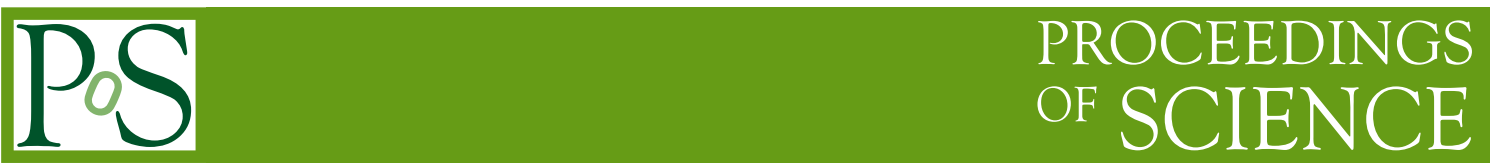

\title{
Is radar detection of extensive air showers feasible?
}

\author{
J. Stasielak ${ }^{* 1}$, R. Engel ${ }^{2}$, S. Baur ${ }^{2}$, P. Neunteufel ${ }^{3}$, R. Šmída² ${ }^{2}$ F. Werner ${ }^{4}$, H. \\ Wilczyński ${ }^{1}$ \\ ${ }^{1}$ Institute of Nuclear Physics PAN, Kraków, Poland \\ ${ }^{2}$ Institute for Nuclear Physics (IKP), Karlsruhe Institute of Technology, Karlsruhe, Germany \\ ${ }^{3}$ Argelander Institute for Astronomy (AIfA), Bonn, Germany \\ ${ }^{4}$ Max-Planck Institute for Nuclear Physics, P.O. Box 103980, D 69029 Heidelberg, Germany \\ E-mail: jaroslaw.stasielak@ifj.edu.pl
}

\begin{abstract}
We investigate the feasibility of the radar technique for extensive air shower detection. A set of simulations of radio wave reflection off the short-lived plasma produced by the high-energy showers in the air is performed, considering various radar setups and shower geometries. We show that the plasma produced by air showers should be treated always as underdense. Thus, we use the Thomson cross-section for scattering of radio waves with correction for molecular quenching. We sum coherently the radio waves reflected off the individual electrons over the volume of the disk-like ionization trail to obtain the time evolution of the signal arriving at the receiver antenna. The movement of the wave-scattering region behind the relativistically moving shower front is taken into account. The received power and the spectral power density of the radar echo are analysed. Based on the obtained results, we conclude that the scattered signal is too weak for the radar method to provide an efficient and inexpensive method of air shower detection. We discuss possible uncertainties of this result.
\end{abstract}

The 34th International Cosmic Ray Conference,

30 July- 6 August, 2015

The Hague, The Netherlands

\footnotetext{
*Speaker.
} 


\section{Introduction}

The remote sensing of extensive air showers (EAS) using a radar system is considered to be a promising technique, which in principle could allow the construction of cosmic ray observatories with very large apertures to be built at low cost. In this technique a ground-based radio transmitter irradiates the ionization trail left behind the shower front and another ground-based antenna receives the scattered radio signal.

There have been several unsuccessful attempts of EAS detection via radar technique in the past, e.g. [1] - [3]. A currently ongoing radar experiment, working in the forward scattering mode, is the TARA project [4]. It consists of a $54 \mathrm{MHz}$ transmitter at the Telescope Array site and a set of radio receivers located approximately $50 \mathrm{~km}$ away from the transmitter. There have been no shower detections confirmed thus far.

The radar technique has been already used for decades to observe the ionization trails that result from meteors or lightning. These trails are traditionally divided into the underdense and overdense regions, depending on the local characteristic plasma frequency $\omega_{p}$. If the electron density is high enough that the plasma frequency $\omega_{p}$ exceeds the radar frequency $\omega$, i.e. the frequency of the emitted radio wave, then the radio wave is reflected from its surface. Such a region is called overdense. In contrast, if the opposite is true, then the region is underdense and the radio wave can penetrate the ionized region. In such a case the reflections by scattering of the radio wave off individual free electrons has to be considered. The cross-section for the radio wave reflection is much larger for the overdense case.

The ionization trail that is produced in the atmosphere by the passage of the high-energy particles of an extensive air shower, consists of electrons, which are essentially at rest with respect to the surrounding atmosphere. The locally produced plasma decays exponentially. For the plasma densities relevant for EAS and at low altitudes, the deionization process is dominated by the threebody attachment of an electron to an oxygen molecule [5]. The plasma lifetime depends on the air density and changes from about $15 \mathrm{~ns}$ at the sea level to $40 \mathrm{~ns}$ at the altitude of $5 \mathrm{~km}$ [5], which leads to the length of the ionization trail of about $10-40 \mathrm{~m}$. The radial dependence of the plasma density is controlled by the lateral distribution of the shower energy deposition in the air. The electron density is highest at the shower axis and decreases steeply with the distance from it. The diameter of the ionization trail is of several hundred meters. The shower front moves approximately with the speed of light in vacuum. Due to the short lifetime of the created plasma, the plasma-filled region behind the shower front also moves with the speed of light even though the electrons of the plasma do not move on macroscopic scales. Therefore, a Doppler effect will be observed in the radar echo, unless the shower is seen from the side. An enhancement of the signal scattered backwards due to its time compression is also expected [6].

In the literature, there are several theoretical approaches to the problem of the EAS radar detection utilizing different modes of detection [6] - [13]. Here, we present the most complete approach yet to the radar detection of air showers by backscattering. However, the results also apply to a bistatic radar setup, where the transmitter and receiver are separated by a distance comparable to the distances of the detector antennas to the shower.

We investigate the feasibility of detecting extensive air showers by the radar technique. Simulations are performed for the underdense regime using the Thomson cross-section for scattering 


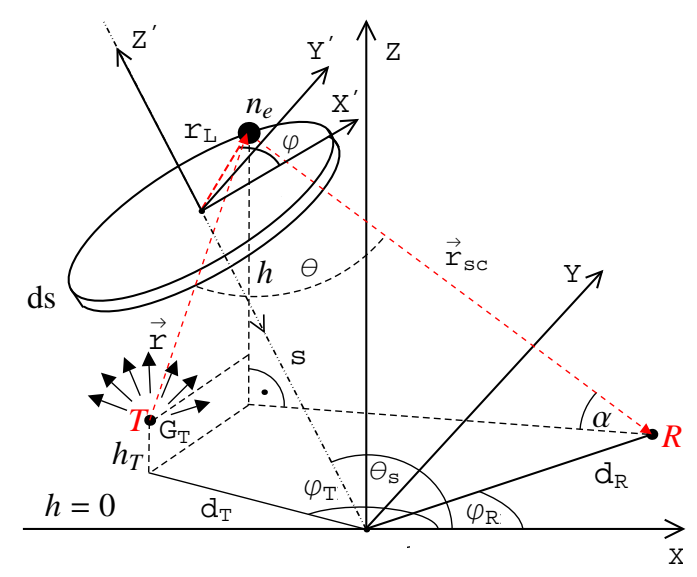

Figure 1: Schematic diagram representing the considered radar system and reflection from an element of the static plasma produced by a shower in the atmosphere. See the text for a detailed explanation.

of radio waves off the short-lived, non-moving plasma, with a correction for molecular quenching. We sum coherently the reflected radio waves off the individual electrons over the volume of the disk-like ionization trail and obtain the time evolution of the radar echo.

\section{Calculation of the radar signal}

A schematic diagram representing the concept of EAS detection using the radar technique is shown in Figure 1. A ground-based radio transmitter (T) irradiates a short-lived, disk-like, nonmoving plasma left behind the shower front. The radio signal is scattered by free electrons in the ionization trail and subsequently received by the ground-based antenna (R). The geometry of such a radar system is described conveniently by the cylindrical coordinates of the transmitter and the receiver, i.e. by the distances from the shower core to the transmitter $\left(d_{T}\right)$ and to the receiver $\left(d_{R}\right)$, and by the angles $\varphi_{T}$ and $\varphi_{R}$.

The disk in Figure 1 represents a slice of the static plasma, whose distance to the shower core at ground is equal to $\mathrm{s}$. Let us consider the plasma volume element $\mathrm{d} V=r_{L} \mathrm{~d} r_{L} \mathrm{~d} \varphi \mathrm{d} s$ with polar coordinates $r_{L}$ (distance from the shower axis) and azimuth $\varphi$. Its contribution to the radar echo at the receiver at time $t$ is, following [14]

$$
\frac{\mathrm{d} U\left(t, s, r_{L}, \varphi\right)}{\mathrm{d} V}=\sqrt{\frac{G_{T}}{4 \pi}} \frac{\sqrt{\Delta \Omega_{s c}}}{|\mathbf{r}|} \sqrt{\left(\frac{\omega}{v_{c}}\right)^{2} \frac{d \sigma_{T}}{d \Omega}} n_{e} e^{i\left(\omega t+\phi_{0}\right)} e^{-i \int_{\mathbf{r}} n \mathbf{k} \cdot \mathrm{dr}} e^{-i \int_{\mathbf{r s c}} n \mathbf{k}_{\mathbf{s c}} \cdot \mathrm{d} \mathbf{r}_{\mathbf{s c}}},
$$

where $G_{T}$ is the transmitter gain factor, $\Delta \Omega_{s c}$ is the solid angle of the receiver as seen from the point of scattering, $\omega=2 \pi v$ is the radian frequency of the emitted radio wave, $v_{c}$ is the collision frequency of an electron with neutral molecules, $\mathrm{d} \sigma_{T} / \mathrm{d} \Omega$ is the differential Thomson cross-section, $n_{e}$ is the electron density of the considered plasma element at the time of reflection, $\phi_{0}$ is the initial phase of the emitted signal, $\mathbf{k}$ and $\mathbf{k}_{\mathbf{s c}}$ are the wave vectors of the incoming and scattered radio wave, and $n$ is the refractive index of the air derived from the U.S. Standard Atmosphere model. The factor $\left(\omega / v_{c}\right)^{2}$ is introduced to take into account the molecular quenching (see Section 3 ).

For large $\left|\mathbf{r}_{\mathrm{sc}}\right|$ we get the approximation $\sqrt{\Delta \Omega_{s c}} \sim \sqrt{A_{R}} / \mathbf{r}_{\mathrm{sc}}$, where $A_{R}$ is the effective area of the receiver antenna, so the field strength of the reflected radio wave diminishes like $|\mathbf{r}|^{-1}\left|\mathbf{r}_{\mathbf{s c}}\right|^{-1}$. 
This is equivalent to an $|\mathbf{r}|^{-2}\left|\mathbf{r}_{\mathbf{s c}}\right|^{-2}$ dependence in the received power, thus the strongest signal is usually obtained from altitudes close to the ground level.

The signal received by the antenna at a given time is a sum of the signals scattered at different times, from different parts of the plasma disk, and from different altitudes. These individual contributions interfere with each other and only an integral over the whole volume $V(t)$ from which they arrive simultaneously gives us the correct value. This volume is, in general, time-dependent and it can extend over a wide range of altitudes (even several kilometers due to the time compression of the received signal). The instantaneous signal strength of the radar echo at the receiver antenna can be expressed by

$$
U(t)=\int_{V(t)} \frac{\mathrm{d} U\left(t, s, r_{L}, \varphi\right)}{\mathrm{d} V} \mathrm{~d} V .
$$

It is a useful dimensionless quantity which facilitates deriving the maximum of the received power. Its real part defines the waveform $R(t)$, which is proportional to the electric field strength at the receiver. The ratio of the instantaneous power $P_{R}(t)$ received by the detector antenna to the power emitted by the transmitter $P_{T}$ is equal to $P_{R}(t) / P_{T}=R^{2}(t)$.

The shape of the waveform $R(t)$ and thus of the received power $P_{R}(t)$ depend, via the electric field, on the initial phase $\phi_{0}$ of the transmitter. Hence, a different choice of the $\phi_{0}$ value will change both the moment at which the detector observes the maximum power and the maximum value of this power $P_{R, m}$. For each shower, we scan through all possible $\phi_{0}$ values to maximize $P_{R, m}$. Let us denote this maximum value of $P_{R, m}$ by $P_{R, \max }$. The ratio of the maximum received power to the emitted power is equal to the square of the maximum value of the function $|U(t)|$, i.e.

$$
P_{R, \max } / P_{T}=\operatorname{Max}\left[|U(t)|^{2}\right] .
$$

\section{Scattering off the plasma}

The electron density of the plasma, produced by the high-energy shower particles in the air, is estimated using the average longitudinal profile of proton showers parametrized by the GaisserHillas function and assuming the Góra function [15] as the lateral distribution. We assume that each shower particle deposits on average $2.3 \mathrm{MeV}$ per traversed $\mathrm{g} / \mathrm{cm}^{2}$ and that all of the deposited energy goes into ionization. The mean energy per ion-pair production is $33.8 \mathrm{eV}$. Note that while the maximum particle count of the shower is described by the Gaisser-Hillas function, the plasma density depends also on the local atmospheric density. Therefore, the point of the highest plasma density is up to several hundred meters below the shower maximum [16].

For a tenuous plasma (with $n_{e}<10^{8} / \mathrm{cm}^{3}$ ), which we consider, the density of free electrons is much lower than the density of neutral molecules. Therefore, the collision frequency $v_{c}$ is dominated by electron collisions with neutral molecules. The value of $v_{c}$ is of high importance in determining scattering properties of the plasma. It can be calculated by appropriately averaging the momentum transfer cross-section for electron impact on $N_{2}$ and $O_{2}$ molecules over the velocity distribution of the plasma electrons. We apply the initial electron distribution, i.e. the velocity distribution soon after the plasma is created by the passing of the shower front [16]. However, as was shown in [17], the energy distribution of the plasma electrons changes very rapidly with time due to the ionization and excitation processes. After $1 \mathrm{~ns}$ all electrons have energies below $1.7 \mathrm{eV}$. 


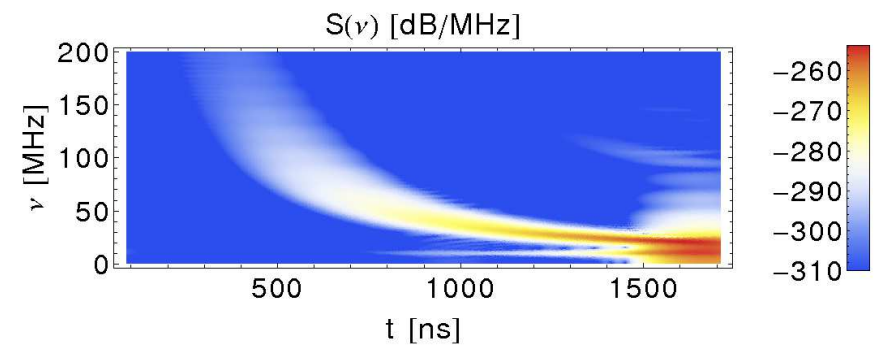

Figure 2: Spectrogram of the radar echo of a vertical shower with energy $10^{18} \mathrm{eV}$ heading towards the transmitter. The radar frequency and transmitter-receiver distance are equal to $v=10 \mathrm{MHz}$ and $d_{R}=500$ $\mathrm{m}$, respectively. A $500 \mathrm{~ns}$ running time window is used.

Nevertheless, this spectrum evolution leads to only relatively small changes in the $v_{c}$ value and up to about a $9 \mathrm{~dB}$ increase in the received power. This does not change the conclusion of our work. The collision frequency $v_{c}$ is of the order of THz.

For a collisionless plasma $\left(v_{c}=0\right)$, the total reflection from the interface between the air and the homogeneous plasma occurs when $\omega \leq \omega_{p}$, and the reflected power drops for $\omega>\omega_{p}$. If collisions are present, then the radio wave is not totally reflected, even for $\omega<\omega_{p}$, and the wave can penetrate the plasma volume. The reflected power is greatly reduced as the electron collision frequency $v_{c}$ increases [18]. For the specular reflection to occur, it is necessary not only that the radio wave frequency $\omega$ is lower than the plasma frequency $\omega_{p}\left(\omega<\omega_{p}\right)$ but also that it is much higher than the collision frequency $v_{c}\left(\omega \gg v_{c}\right)$. In our case, these conditions are not fulfilled. Even though the first condition might be met by the plasma produced by the shower at its axis (for a low radar frequency, i.e. $v<16 \mathrm{MHz})$, the second one will not be fulfilled $\left(v_{c} \sim \mathrm{THz}\right)$.

It follows that we can not use an analogy with the reflective behaviour of the overdense ionization trails produced by meteors high in the atmosphere and apply it to the plasma produced by the shower at much lower altitudes. An overdense meteor trail can be modeled as a thin metallic cylinder and the corresponding radar cross-section can be used. In contrast, the plasma induced by a shower has to be treated as underdense in all cases. The radio wave can penetrate the ionized region and the reflections of the radio wave are caused by its scattering on individual electrons. The total scattered signal is a sum of these individual contributions.

The Thomson cross-section $\sigma_{T}$ describes scattering of the electromagnetic wave on a nonrelativistic, free electron in vacuum, i.e. when there are no collisions with other particles. The net effect of the electron collisions with neutral molecules is a reduction of the electron acceleration in the radio wave field and thus a decrease in the power re-radiated by the electron. It can be shown that for $v_{c} \gg \omega$ the cross-section for radio wave scattering is reduced by the factor $\left(\omega / v_{c}\right)^{2}$ [14]. This effect is called the molecular quenching and is responsible for a decrease in the power recorded in the receiver antenna by several orders of magnitude.

\section{Results}

An extensive set of simulations of radar detection of air showers were made. The simulations were performed assuming that the effective area of the receiver antenna is $A_{R}=1 \mathrm{~m}^{2}$ and the groundbased transmitter emits a signal isotropically into the whole upper hemisphere (i.e. $G_{T}=2$ ). We 

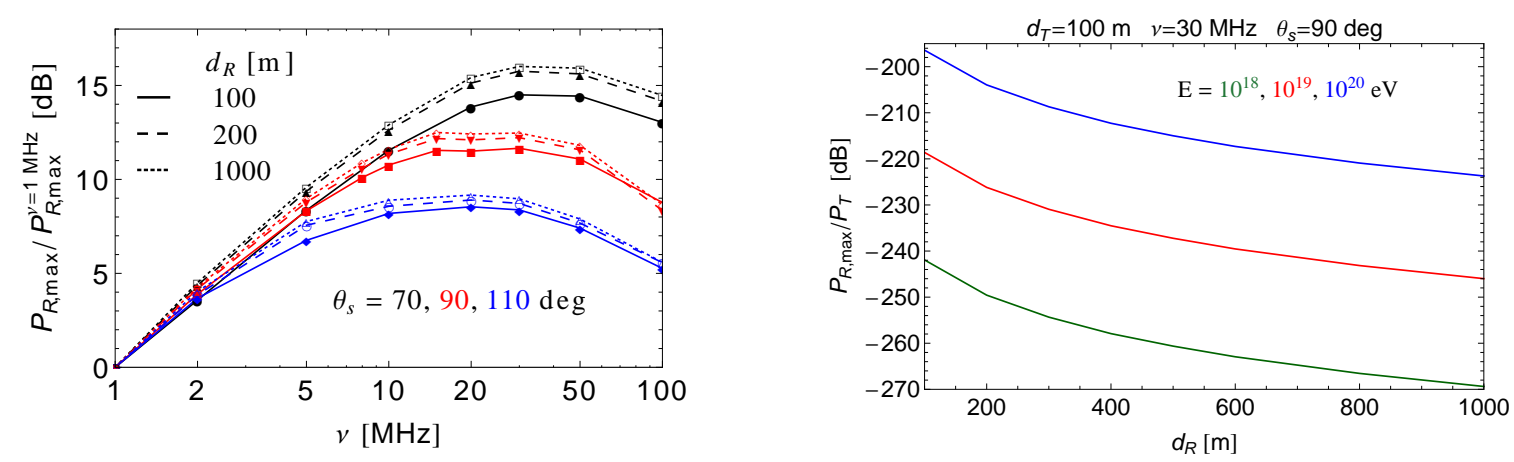

Figure 3: (left panel) Dependence of the maximum received power on the radar frequency $v$ and the angle $\theta_{s}$ (see Figure 1), scaled to the respective maximum power received for frequency of $1 \mathrm{MHz}$. The case of showers heading towards the transmitter is considered here $\left(d_{T}=0\right)$. (right panel) Ratio of the maximum received to the emitted power as a function of the shower core-receiver distance $d_{R}$ and shower energy $E$, for vertical showers at $100 \mathrm{~m}$ from the transmitter. The radar frequency is $30 \mathrm{MHz}$.

also assume that the receiver points vertically upward and the solid angles at which it is seen from each point of scattering are taken into account. Thus, we are not constrained to the far field approximation. Both the transmitter and the receiver are located at sea level. We analyze only the signal which arrives to the receiver antenna. We do not consider any characteristics of the antenna, i.e. the receiver is assumed to be ideal and its efficiency is independent of the frequency of the radar echo.

An example of spectrogram of the radar echo is shown in Figure 2. A vertical shower with energy $10^{18} \mathrm{eV}$ heading towards the transmitter $\left(d_{T}=0\right)$ is considered here. The radar frequency and transmitter-receiver distance are equal to $v=10 \mathrm{MHz}$ and $d_{R}=500 \mathrm{~m}$, respectively. The frequency of the radar echo decreases with time. The typical signal consists of two parts: a short signal upshifted to high-frequency with low amplitudes and a long signal with frequency upshifts of only a few and larger amplitudes.

We have calculated the maximum received power $P_{R, \max }$ for each of the simulated radar echoes. The dependence of $P_{R, \max }$ on the shower energy shows a universal scaling. There is about $23 \mathrm{~dB}$ increase in power per decade increase in shower energy. This scaling is similar to that in coherent scattering, in which the reflected power depends quadratically on the number of scattering particles.

The left panel of Figure 3 shows the dependence of the maximum received power on the radar frequency $v$ and the angle $\theta_{s}$ (see Figure 1) scaled to the respective maximum power received at the frequency of $1 \mathrm{MHz}$. The size of the region, from which one gets a coherent signal, decreases with decreasing wavelength and destructive interference cancels out the signal from the farther regions of the plasma. At large frequencies, this leads to a decrease in the received power $P_{R, \max }$ with increasing radar frequency $v$. On the other hand, $P_{R, \max }$ scales as $\left(\omega / v_{c}\right)^{2}$ due to the molecular quenching. Therefore, the received power is quenched more for the lower radar frequencies. Effectively, the strongest signal is reached at frequencies around $30 \mathrm{MHz}$. For vertical showers, the received power increases by about $12 \mathrm{~dB}$ when changing the radar frequency from $1 \mathrm{MHz}$ to 30 $\mathrm{MHz}$. This increase is larger for inclined showers. However, this does not translate to the larger values of $P_{R, \max }$, since in general the inclined showers give weaker signals.

The right panel of Figure 3 shows the dependence of the received power on the shower core- 
receiver distance $d_{R}$ and shower energy $E$, for vertical showers at $100 \mathrm{~m}$ from the transmitter. The radar frequency is $30 \mathrm{MHz}$. The shown values of the power ratio $P_{R, \max } / P_{T}$ represent the strongest signal that can be attained in the $\mathrm{MHz}$ frequency range for $d_{T}$ and $d_{R}$ larger than $100 \mathrm{~m}$. The largest value of the power ratio $P_{R, \max } / P_{T}$ is equal to $-196 \mathrm{~dB}$ for $d_{T}=d_{R}=100 \mathrm{~m}$ and for highest energy $E=10^{20} \mathrm{eV}$. The frequency upshift $f_{r}$ of the received signal at its maximum is small, typically $f_{r}=$ $2-3$. Thus, the radar echoes of air showers can be observed best at frequencies $v_{R}=60-90 \mathrm{MHz}$ ( $v_{R}$ denotes the frequency of the received signal). Within this frequency range, for a location with small man-made noise the noise temperature is dominated by the Galactic synchrotron emission and it changes from about $4400 \mathrm{~K}$ to $2000 \mathrm{~K}$, respectively. It follows that assuming the effective bandwidth of the receiver antenna to be $\Delta v_{R}=10 \mathrm{MHz}$, one gets the power of the noise $P_{n}=$ $k_{B} T_{n} \Delta v_{R} \geq-95.6 \mathrm{dBm}$, where $k_{B}$ is the Boltzmann constant. Imposing a $5 \mathrm{~dB}$ signal-to-noise ratio (SNR) as the detectability threshold leads to the condition $\mathrm{SNR}=P_{R, \max } / P_{n} \geq 5 \mathrm{~dB}$. Let us assume that the effective area of the receiver is as large as $A_{R}=10 \mathrm{~m}^{2}$ and the transmitted power is equal to $P_{T}=10^{5} \mathrm{~W}$. For a grid of $N$ receiving antennas, the signal power scales coherently like $P_{R, \max } \sim N^{2}$, whereas the noise is incoherent and scales linearly, i.e. $P_{n} \sim N$. Therefore, we can get a $10 \mathrm{~dB}$ increase in the signal-to-noise ratio by utilizing a grid of 10 receiving antennas. Moreover, we estimate that by tilting the antennas to the horizontal position we can get the signal increase by about $8 \mathrm{~dB}$ for $d_{R}=100$. As the result, we obtain $\mathrm{SNR}=7.6 \mathrm{~dB}$ for $d_{R}=100 \mathrm{~m}$, which falls down below $5 \mathrm{~dB}$ already around $d_{R}=200 \mathrm{~m}$. This demonstrates that in general, detection of the radar echoes of the $10^{20} \mathrm{eV}$ showers might be possible, however, the necessity of using high-power transmitters, the large number of receivers and the small spacing of the detector grid (of the order of $300 \mathrm{~m}$ ) makes the radar technique utilizing the $\mathrm{MHz}$ range impractical for air shower detection.

We estimate the overall accuracy of our results at about $5 \mathrm{~dB}$. This number does not account for a possible inaccuracy in the assumed plasma lifetime and the collision frequency (molecular quenching), which might have a substantial effect.

\section{Conclusion}

We have performed an extensive set of simulations of radar detection of air showers for different geometries, energies and frequencies of the radar. We have shown that, unlike the plasma in a meteor trail, the plasma produced by air showers has to be treated always as underdense and the reflected radar signal is heavily reduced by molecular quenching. We have summed coherently the radio waves reflected off the individual electrons over the volume of the disk-like ionization trail, taking into account the movement of the wave scattering region behind the relativistically moving shower front.

Our results show that the radar detection of air showers might be possible only in a special case of showers at small zenith angles with energies $10^{20} \mathrm{eV}$ or higher and shower cores situated very close to the transmitter and receiver antennas. It appears therefore that the radar technique of air showers detection on a large scale is impractical due to the necessity to use very high power transmitters and very small spacing of the detector grid. Thus, developing large, efficient and inexpensive air shower detector arrays based on the radar technique is not realistic. 


\section{Acknowledgment}

This work has been supported in part by the National Centre for Research and Development (NCBiR) grant ERA-NET-ASPERA/01/11, the ASPERA project BMBF 05A11VKA and the KIT start-up grant 2066995641.

\section{References}

[1] T. Matano et al., Tokyo large air shower project, Can. J. Phys. 46 (1968) S255.

[2] T. Vinogradova et al., Proposed experiment to detect air showers with the Jicamarca radar system, AIP Conf. Proc., 579 (2001) 271.

[3] D. Wahl, J. Chau, J. Bellido, The search for vertical extended air shower signals at the Jicamarca Radio Observatory, Proc. of the 30th ICRC, Merida, Mexico 5 (2007) 957.

[4] R. Abbasi et al., Telescope Array Radar (TARA) Observatory for Ultra-High Energy Cosmic Rays, Nucl. Instrum. Meth. Phys. Res. A 767 (2014) 322, [arXiv:1405.0057].

[5] S. Nijdam et al., Probing background ionization: positive streamers with varying pulse repetition rate and with a radioactive admixture, J. Phys. D: Appl. Phys. 44 (2011) 455201.

[6] J. Stasielak et al., Enhancement of the radar signal of air showers due to time compression, Proc. of the 33rd ICRC, Rio de Janeiro (2013), paper 0473, [arXiv:1310.0743].

[7] P. W. Gorham, On the possibility of radar echo detection of ultra-high energy cosmic ray-and neutrino-induced extensive air showers, Astropart. Phys. 15 (2001) 177.

[8] M. I. Bakunov et al., Relativistic effects in radar detection of ionization fronts produced by ultra-high energy cosmic rays, Astropart. Phys. 33 (2010) 335.

[9] M. I. Bakunov et al., Prospects for radar detection of cosmic ray air showers with medium-frequency radio waves, New J. Phys. 15 (2013) 113027.

[10] H. Takai et al., Forward Scattering Radar for Ultra High Energy Cosmic Rays, Proc. of the 32nd ICRC, Beijing 3 (2011) 344.

[11] J. Stasielak et al., Radar reflection off extensive air showers, EPJ Web of Conferences 53 (2013) 08013, [arXiv:1210.1427].

[12] A. D. Filonenko, Coherent scattering of monochromatic $R F$ radiation by ionization electrons of an extensive air shower, J. Exp. Theor. Phys. 117 (2013) 641.

[13] K. D. de Vries, K. Hanson, T. Meures, On the feasibility of RADAR detection of high-energy neutrino-induced showers in ice, Astropart. Phys. 60 (2015) 25, [arXiv:1312.4331].

[14] J. Stasielak et al., Feasibility of radar detection of extensive air showers, arXiv:1411.7295.

[15] D. Góra et al., Universal lateral distribution of energy deposit in air showers and its application to shower reconstruction, Astropart. Phys. 24 (2006) 484, [arXiv:0505371].

[16] P. Neunteufel et al., Microwave Emission due to Molecular Bremsstrahlung in Non-Thermal Air Shower Plasmas, Proc. of the 33rd ICRC, Rio de Janeiro (2013), paper 1220.

[17] I. Al Samarai et al., An Estimate of the Spectral Intensity Expected from the Molecular Bremsstrahlung Radiation in Extensive Air Showers, arXiv:1409.5051.

[18] J. Santoru, \& D. J. Gregoire, Electromagnetic-wave absorption in highly collisional plasmas, J. Appl. Phys. 74 (1993) 3736. 